\title{
MICHAËL WYSS
}

\section{APPORT DES RECHERCHES ARCHÉOLOGIQUES RÉCENTES POUR LA CONNAISSANCE DE SAINT-DENIS AUX XI ${ }^{\mathrm{e}}$ ET XII ${ }^{\mathrm{e}}$ SIÈCLES}

\begin{abstract}
À l'échelle de l'histoire presque bimillénaire de Saint-Denis, la période concernée est un moment décisif, celui qui a vu l'éclosion d'un bourg monastique aux portes de la célèbre abbaye bénédictine. Si les rares sources écrites peuvent nous informer sur les facteurs spirituels, politiques et économiques qui ont présidé à ce phénomène, il revient à l'archéologie de nous faire connaître les modalités concrètes de cette évolution. Par chance, depuis près de trente ans, le patrimoine archéologique de Saint-Denis fait l'objet d'une enquête systématique. De 1973
\end{abstract} à 1992, la rénovation urbaine du quartier situé au nord de la basilique donna l'occasion d'effectuer une fouille d'envergure, portant sur plus de treize hectares de la ville médiévale. Plus récemment, les travaux d'aménagement des abords immédiats de la basilique ont pu être mis à profit pour compléter notre documentation sur le centre monumental. L'exploitation scientifique des données de fouille étant toujours en cours, ces quelques lignes reflètent l'état actuel de nos connaissances qui ne préjuge en rien des résultats définitifs qu'apportera l'achèvement de ce travail'.

Le haut Moyen Âge a doté le site d'un complexe monumental, centré sur l'église abbatiale, et d'un ouvrage de défense, le castellum (fig. 1). Il nous faut évoquer brièvement ces deux composantes, car la topographie si caractéristique du bourg, au réseau de rues radio-concentriques, montre du premier coup d'œil l'incidence qu'eurent le noyau monumental et le rempart sur l'organisation spatiale de l'agglomération.

L'abbatiale carolingienne, connue grâce aux fouilles de Sumner McKnight Crosby ${ }^{2}$, était une construction que l'abbé Fulrad (vers 750-784) avait conçue sur

1 Les fouilles, menées en prévision des graves atteintes au sous-sol occasionnées par les travaux de reconstruction du centre-ville, furent conduites par Olivier Meyer et durèrent de 1973 à 1992. Depuis la fin des opérations, une partie de l'équipe municipale (Unité d'Archéologie de la Ville de Saint-Denis [UASD]), dirigée par Nicole Meyer-Rodrigues, est mobilisée par l'exploitation de la masse documentaire recueillie en vingt années de travaux de terrain. En 1996, le programme de publication a pu être inauguré avec la parution de: Michaël WYSS (dir.), Atlas historique de Saint-Denis: des origines au XVIII ${ }^{e}$ siècle, Paris 1996 (Documents d'archéologie française, 59).

2 Sumner McKnight CROSBY, The Royal Abbey of Saint-Denis from Its Beginnings to the Death of Suger, 475-1151, éd. par Pamela Z. BLUM (Yale Publications in the History of Art, 
le plan d'une basilique à colonnes dont le transept ouvrait, à l'est, sur une abside semi-circulaire. À l'ouest, l'édifice était précédé d'un massif occidental assimilé par Suger à l'augmentum que Charlemagne aurait fait édifier sur la tombe de son père Pépin, mis en terre ante limina basilicae ${ }^{3}$. L'église comportait deux portes latérales donnant, au sud, sur les bâtiments communautaires et, au nord, sur la nécropole, devenue grand cimetière ou aitre de l'abbaye.

Dès l'époque mérovingienne, l'aire funéraire était bordée d'au moins trois églises cimétériales (Saint-Barthélemy, Saint-Pierre, Saint-Paul) reliées entre elles par des annexes et des galeries. Ce cadre architectural n'est pas sans faire penser à l'atrium mentionné dans les sources contemporaines. Devant l'entrée de l'abbatiale, une zone résidentielle a atteint son apogée sous la forme d'un important bâtiment d'apparat que nous proposons d'identifier au palatium attesté à Saint-Denis sous les Carolingiens ${ }^{4}$.

À partir de la fin du IX ${ }^{e}$ siècle, le centre monumental subit de profonds remaniements. Les églises cimétériales voient leurs annexes supprimées, modification architecturale à mettre en rapport avec leur probable affectation aux paroisses du bourg. La construction du palais, bien qu'à l'architecture de pierre, est également abandonnée et, dès le $\mathrm{XI}^{\mathrm{e}}$ siècle, une partie de sa ruine est rasée pour laisser place à l'extension du cimetière. Parallèlement, la séparation entre l'aire funéraire et la zone d'habitat civil se matérialise sous la forme d'un imposant mur de clôture.

Rolf Große vient de faire le point sur la question des origines du collège des chanoines de Saint-Paul ${ }^{5}$. L'établissement de la communauté dans cette église cimétériale semble être une conséquence des mesures de réforme de l'abbaye que Robert II le Pieux confia à l'abbé clunisien Odilon. Pour l'heure, les fouilles ont seulement porté sur la première travée ouest et les abords nord de la collégiale; mais on a pu constater, qu'au $\mathrm{XI}^{\mathrm{e}}$ siècle, cette église se distingue déjà par un plan

37), New Haven, Londres 1987.

3 Epistola ad Hilduinum, éd. par Ernst DUMMLER, dans: MGH Epistolae, t. 5, Berlin 1890, p. 326. Le roi demande à être mis en terre à l'extérieur de la basilique. Suger, De administratione, éd. par Françoise GASPARRI, Suger, Euvres, t. 1, Paris 1996, p. 112. L'abbé identifie l'augmentum de Charlemagne. À propos des vestiges matériels de cette construction voir: Werner JACOBSEN, Michaël WYSS, Saint-Denis: essai sur la genèse du massif occidental, dans: Christian SAPIN (dir.), Avant-nefs et espaces d'accueil dans l'église entre le IV et le XII siècle: actes du colloque international du CNRS, Auxerre 17-20 juin 1999, Paris 2002, p. 7687.

4 Michaël WYSS, Die Klosterpfalz Saint-Denis im Licht der neuen Ausgrabungen, dans: Deutsche Königspfalzen. Beiträge zu ihrer historischen und archäologischen Erforschung, t. 5 (Veröffentlichungen des Max-Planck-Instituts für Geschichte, 11), Göttingen 2001, p. 175192; D., Un établissement carolingien mis au jour à proximité de l'abbaye de Saint-Denis: la question du palais de Charlemagne, dans: Annie RENOUX (dir.), »Aux marches du palais«. Qu'est-ce-qu'un palais médiéval? Données historiques et archéologiques: actes du VII Congrès international d'Archéologie Médiévale, Le Mans - Mayenne 9-11 septembre 1999, Le Mans 2001, p. 191-200.

5 Rolf GrosSE, Saint-Denis zwischen Adel und König. Die Zeit vor Suger, 1053-1122 (Beihefte der Francia, 57), Stuttgart 2002, p. 201-205. 
basilical: une nef à collatéraux dont la largeur extérieure de $16,50 \mathrm{~m}$ était proportionnée à sa longueur, estimée à $40 \mathrm{~m}$. En provient, probablement, un chapiteau (fig. 2) réemployé à l'Époque moderne dans la maison du chantre de Saint-Paul, sise immédiatement au nord de l'église ${ }^{6}$. La pièce, taillée dans un calcaire grossier, est ornée d'un rinceau de palmettes et d'un masque à large gueule. D'après le style, la datation de ce chapiteau se situe dans la deuxième moitié du $\mathrm{XI}^{e}$ siècle. La construction de ce premier état de la collégiale alla de pair avec une profonde réorganisation de l'espace observée en fouille dans l'enclos (claustrum) attenant l'église au nord.

Entre 1135 environ et 1144, l'abbé Suger agrandit l'abbatiale par un nouveau massif occidental et un chœur à chapelles rayonnantes. Avec l'ouverture des trois portails, la construction souligne le rôle d'entrée de la nouvelle façade. Dans le cimetière, l'édification d'une église, placée sous le vocable de saint Michel, témoigne amplement de cette mise en chantier du centre monumental. L'édifice est de plan très simple: une nef unique qui ouvre sur une abside rectangulaire. Adossé à la face interne de la clôture du cimetière, il s'insère dans la série des églises Saint-Barthélemy et Saint-Pierre, survivances de l'époque antérieure. La construction réemploie dans ses fondations un ensemble d'éléments d'architecture et de décors sculptés attribuables au premier tiers du XII ${ }^{\mathfrak{e}}$ siècle et provenant probablement d'une partie de la basilique alors en reconstruction ${ }^{7}$. Le chapiteau d'une colonne libre est orné des scènes de la parabole du Mauvais Riche et de Lazare (fig. 3). De par ses dimensions, ce chapiteau a très bien pu s'adapter aux colonnes de l'abbatiale carolingienne qui, au bout de près de trois cents ans d'existence, a certainement eu besoin d'être restaurée. Une série de chapiteaux plus petits, se rapporte plus probablement à un cycle hagiographique. Cette hypothèse est appuyée par une scène qui semble représenter le moment où Denis et ses deux compagnons sont conduits devant le prévôt Sisinnius (fig. 4).

Vers le milieu du XII ${ }^{\mathrm{e}}$ siècle, la réédification des bâtiments communautaires commence par la construction d'un nouveau cloître. C'est en effet au décor sculpté des galeries de ce cloître que l'on attribue plusieurs chapiteaux et une statue-colonne qui se trouvent aujourd'hui dispersés dans les musées d'Europe et des États-Unis ${ }^{8}$. D'autres fragments recueillis en fouille et les éléments d'une

6 Danielle JOHNSON, Recherche sur l'origine d'un chapiteau en calcaire découvert à SaintDenis, dans: Jacqueline LORENZ, Paul BENOIT, Daniel OBERT (dir.), Pierres et carrières. Géologie - Archéologie - Histoire: actes des journées Claude Lorenz, Paris 17 et 18 novembre 1995, Paris 1997, p. 221-224.

7 Michaël WYSS, Saint-Denis, I: sculptures romanes découvertes lors des fouilles urbaines, dans: Bulletin monumental 150 (1992), p. 309-354.

8 Léon PRESSOUYRE, Did Suger build the Cloister at Saint-Denis?, dans: Paula Lieber GERSON (dir.), Abbot Suger and Saint-Denis. A Symposium, New York 1986, p. 229-244. 
arcature aux écoinçons ornés (fig. 5) concordent avec cette construction' qui, en 1750, fut livrée aux pioches des démolisseurs.

Le castellum, fortification du monastère commanditée en 869 par Charles le Chauve $^{10}$, est aujourd'hui documenté, non seulement par les sources archivistiques mais également par l'archéologie. Les fouilles ont porté sur plusieurs tronçons des fossés d'eau et sur l'une des quatre portes d'accès, connue au Moyen Âge sous le nom de porte de la Boucherie. Le dispositif qui protégeait un espace d'environ huit hectares, était irrigué par le Croult, un canal qui amenait les eaux d'une rivière sur plus de six kilomètres. Les fossés étaient doublés, intérieurement, d'une levée de terre, renforcée par un mur, et d'une chaussée empierrée faisant office de chemin de ronde. Le mur du castellum est cité dans les sources, jusqu'au XIII siècle, quant aux portes, elles ont fonctionné jusqu'à l'Époque moderne.

Dès le $X^{\mathfrak{e}}$ siècle les fossés s'envasent, mais le cours d'eau est conservé, endigué par des palissades soigneusement entretenues. En tant qu'équipement hydraulique, cette rivière a certainement joué un rôle moteur dans l'essor du bourg.

L'agglomération civile s'étendait depuis le centre monumental jusqu'au rempart du castellum $^{11}$. Quant à son organisation, la fouille atteste surtout une grande mobilité dans l'utilisation de l'espace. À la différence du tracé, relativement stable, du réseau routier étroitement lié au castellum - rues menant aux portes et chemin de ronde -, les cheminements secondaires fluctuent jusqu'au XIIr' siècle. C'est seulement à ce moment-là que l'occupation de l'espace se fixe sous la forme d'un réseau de petites parcelles.

Trous de poteaux et tranchées de sablières témoignent d'habitations à l'architecture de bois; une pièce de bois creusée de deux rainures atteste la technique de l'assemblage bouveté propre aux constructions de type Stabbau (fig. 6). Les plans des maisons sont malheureusement trop lacunaires pour que nous puissions en proposer une reconstitution. Les sols intérieurs étaient en terre battue et les cloisons faites de branches souples entrelacées, recouvertes d'un enduit de plâtre. Des bardeaux de chêne, percés de trous de chevilles, proviennent de la couverture des toits.

Plusieurs types de structures annexes sont associés à ces maisons. Les fonds de

9 Danièle JOHNSON, Michaël WYSS, Saint-Denis, II: sculptures gothiques récemment découvertes, dans: Bulletin monumental 150 (1992), p. 355-381; Nicole MEYER-RODRIGUES, Michaël WYSS, Nouvelles données archéologiques sur le cloître de l'abbaye de Saint-Denis, dans: „Utilis est lapis in structuraর: Mélanges offerts à Léon Pressouyre, Paris 2000, p. 111-126.

${ }^{10}$ Annales Bertiniani, éd. par Georg WAITZ (MGH SS rer. Germ. in usum schol., 5), Hanovre 1883, p. 98.

" Michaël WYSS, L'agglomération du haut Moyen Âge aux abords de l'abbatiale de Saint-Denis, dans: Hans Rudolf SENNHAUSER (dir.), Wohn- und Wirtschaftsbauten frühmittelalterlicher Klठster: Internationales Symposium, 26.9.-1.10.1995 in Zurzach und Müstair, im Zusammenhang mit den Untersuchungen im Kloster St. Johann in Müstair (Veröffentlichungen des Instituts für Denkmalpflege an der ETH Zürich, 17), Zurich 1996, p. 259-268. 
cabanes ne mesurent guère plus de $3 \mathrm{~m}$ de long sur $2 \mathrm{~m}$ de large; la particularité de ces constructions réside dans le fait qu'elles ont été creusées dans le sol, jusqu'à $1 \mathrm{~m}$ de profondeur. Sur le fond de certaines cabanes on distingue les empreintes laissées par des équipements légers tels que des métiers à tisser.

Les fosses de stockage servaient à la conservation des graines et autres denrées en atmosphère confinée. Après une période d'utilisation que nous estimons relativement courte, ces structures étaient souvent converties en dépotoirs.

La maçonnerie est mise en ouvre pour la construction des puits, mais c'est seulement à partir de la fin du XIII ${ }^{\mathrm{e}}$ siècle qu'elle se généralisera dans l'habitation vernaculaire.

À ce jour, le voisinage du castellum a révélé au moins cinq autres noyaux d'habitations. Les raisons qui ont commandé à leur implantation (présence d'une église, d'une voie de passage, d'un cours d'eau, etc.) nous échappent la plupart du temps. Habituellement leur occupation est réduite dans le temps; on serait donc tenté d'imaginer des établissements tournant autour de l'abbaye suivant une évolution complexe. Plusieurs églises qui relevaient plus ou moins directement de l'abbaye, ont pu subvenir à la gestion spirituelle de ces faubourgs: SaintMartin et le prieuré de l'abbaye, Saint-Denis de l'Estrée, situés tous deux en bordure de la voie antique, Saint-Marcel, aux mains des seigneurs de Montmorency, Saint-Remi et Saint-Quentin (fig. 1).

Les témoins concrets de la vie quotidienne des habitants de Saint-Denis nous sont parvenus sous la forme de ces très grandes quantités d'objets archéologiques qui proviennent essentiellement des fosses-dépotoirs, des alluvions des fossés du rempart et de la rivière urbaine. De tels milieux d'enfouissement, humides et anaérobies, ont favorisé la conservation des matériaux organiques, bois et cuir en particulier; aussi est-ce une image plus complète de la réalité qu'ils nous offrent: écuelles en bois tourné dont le fond est souvent marqué au fer d'une lettre ou d'un motif stylisé ${ }^{12}$, cuillères et peignes parfois gravés d'ocelles, chaussures en cuir. Des clés en fer se rapportent à l'équipement domestique.

En ce qui concerne la céramique, le $\mathrm{XI}^{\mathrm{e}}-\mathrm{XII}^{\mathrm{e}}$ siècle est une période charnière au cours de laquelle vont se développer à la fois la production des poteries à pâte sableuse et paroi fine, et l'emploi de la glaçure plombifere, apparue à Saint-Denis au cours du $X^{\mathrm{e}}$ siècle $^{13}$. L'oule (fig. 7), simple pot destiné à la cuisson et à la conservation, est la forme commune prédominante. À partir du XI ${ }^{\mathrm{e}}$ siècle, elle adopte la lèvre »en bandeau« qui la caractérisera tout au long du Moyen Âge. C'est aussi au cours du $\mathrm{XI}^{\mathrm{e}}-\mathrm{XII}^{\mathrm{e}}$ siècle qu'apparaît le décor peint à l'ocre, dit »en

12 Pierre MLLLE, Les récipients monoxyles: la collection des récipients de table de Saint-Denis, dans: Boire et manger en île-de-France: actes des Journées archéologiques d'île-de-France, Paris 9-10 décembre 2000, Paris 2001, p. 34-37.

${ }^{13}$ Nicole RODRIGUES, Panorama de la poterie francilienne du $\mathrm{XI}^{\mathrm{e}}$ au $\mathrm{XV}^{\mathrm{e}}$ siècle au travers de la collection archéologique de Saint-Denis, dans: L'île-de-France médiévale, t. 1, Paris 2001, p. 82-88. 
flammules« dont l'emploi se généralisera en île-de-France jusqu'à l'Époque moderne. Les cruches à bec verseur tubulaire dont la morphologie générale est héritée de la période carolingienne, accueillent la glaçure. Ces récipients »de luxe«, au décor d'appliques parfois colorées, rehaussées par des motifs estampés, témoignent de l'aisance certaine de leurs utilisateurs; ils seront progressivement remplacés, à partir du XII ${ }^{\mathrm{e}}$ siècle, par des pichets à anses rondes dont la forme s'inspire de pièces métalliques.

Pour appuyer ce constat, on peut également évoquer la présence de verreries qui, pour cette période, se retrouvent malheureusement dans un état d'altération extrêmement critique ${ }^{14}$. L'essentiel du répertoire des formes et des décors procède de la tradition carolingienne. Les deux types les plus courants sont le gobelet globulaire, habituellement orné de filets appliqués en spirale, et le gobelet haut, qui s'évase vers le haut en une coupe.

La découverte, dans un dépotoir du premier quart du XII ${ }^{e}$ siècle, d'un jeu de tables (fig. 8) ${ }^{15}$ est surprenante, puisque cette catégorie d'objets est plutôt associée à des établissements à caractère aristocratique. Notre restitution de son tablier est fondée sur les observations qui ont pu être effectuées au moment du prélèvement des éléments de tabletterie en os, seuls conservés.

L'archéologie met également en lumière les activités artisanales qui ont contribué à construire le bourg monastique. À elle seule, la variété des matériaux mis en œuvre témoigne de la diversité des productions.

Le travail de l'os a dû être fort répandu et principalement fondé sur la récupération des os de boucherie. Quelques coups de couteau suffisent pour transformer un radius de bouf en patin à glace ou un tibia de chèvre en un flageolet. Les fonds de cabanes, précédemment évoqués, sont quasiment les seules traces immobilières de l'activité du tissage. Fuseaux en bois et fusaïoles sont d'autres témoignages de cette production de type domestique.

Mais le cadre spécifique de Saint-Denis se répercute également dans quelques témoignages de l'artisanat spécialisé. Dans le courant du XI ${ }^{e}$ siècle, trois fragments de moules à plombs de vitraux ont été rejetés dans une fosse fouillée, une centaine de mètres au nord de la basilique ${ }^{16}$. Ils étaient associés à des baguettes de plomb et à plusieurs éléments de vitraux peints qui sont peut-être carolingiens. La nature de l'activité reste en suspens: a-t-on fabriqué au $\mathrm{XI}^{\mathfrak{e}}$ siècle de nouvelles verrières, ou en a-t-on simplement réparé d'anciennes? En tout état de cause, la découverte atteste de l'existence d'un atelier, temporaire ou permanent, bien

${ }^{14}$ Nicole MEYER, La verrerie de Saint-Denis, dans: À travers le verre, du Moyen Âge à la Renaissance, Rouen 1989, p. 370-381.

${ }^{15}$ Michaěl WYSS, Ein Spielbrett des 12. Jahrhunderts aus Saint-Denis, dans: Antje KLUGEPINSKER (dir.), Schach und Trictrac. Spielfreude in salischer Zeit, Sigmaringen 1991, p. 5861; Nicole MEYER, Michaël WYSS, Un jeu de tables du XII ${ }^{e}$ siècle provenant de Saint-Denis, dans: Archéologie médiévale 21 (1991), p. 103-113.

${ }^{16}$ Nicole MEYER, Michaël WYSS, Des moules à plombs découverts à Saint-Denis, dans: Bulletin monumental 149 (1991), p. 104-106. 
avant que Suger n'institue son fameux office de maître verrier chargé de l'entretien des vitraux de la basilique ${ }^{17}$.

Au siècle suivant, dans une habitation implantée en bordure du parvis de l'abbatiale, en la place Panetière, la quantité de scories en alliage cuivreux abandonnée dans les niveaux de sol intérieur indique l'activité d'un probable fondeur de bronze. Dans un dépotoir qui en dépend nous avons prélevé les fragments d'un objet très oxydé qui, après un patient travail de restauration, s'est révélé être un chandelier en bronze (fig. 9) ${ }^{18}$. La pièce, haute de $41 \mathrm{~cm}$, est constituée d'un socle ajouré, d'une tige à trois nœuds sphériques et d'une bobèche. Au-dessus de chaque pied du socle émerge une tête de lion, ou de dragon, à long cou, motif qui caractérise une série de chandeliers liturgiques tels que ceux de Kremsmünster, datés du $\mathrm{XI}^{\mathfrak{e}}$ siècle, voire du siècle précédent.

Il est probable que le chandelier soit passé par l'officine du fondeur mais nous ignorons par quel concours de circonstances il a été abandonné. Cette objet, très exceptionnel en fouille, pose une nouvelle fois le problème du cadre de cette production. À n'en pas douter, l'activité du bourg monastique fut orientée vers l'abbaye. Mais comment s'est-elle distinguée des ateliers que l'abbaye faisaient travailler en gestion directe? En incitant des artisans spécialisés, tel que Robert l'Orfêvre ${ }^{19}$, à s'installer près du monastère, l'abbaye a stimulé la vie économique du bourg. Progressivement, une élite urbaine s'est substituée à l'ancien corps aristocratique qui gravitait autour de l'abbaye royale carolingienne.

${ }^{17}$ Louis GrODECKI, Les vitraux de Saint-Denis: étude sur le vitrail au XII ${ }^{e}$ siècle, Paris 1976, p. 28.

${ }^{18}$ Nicole MEYER-RODRIGUES, Un chandelier médiéval en bronze découvert à Saint-Denis, dans: Bulletin de la Société Nationale des Antiquaires de France (1993), p. 71-78.

${ }^{19}$ Confirmation, par Suger, de la vente faite par Girard l'Hospitalier à Robert l'Orfevre, d'un terrain sis devant le monastère, éd. par Françoise GASPARRI, Suger, Guvres, t. 2, Paris 2001, p. $180-183$. 


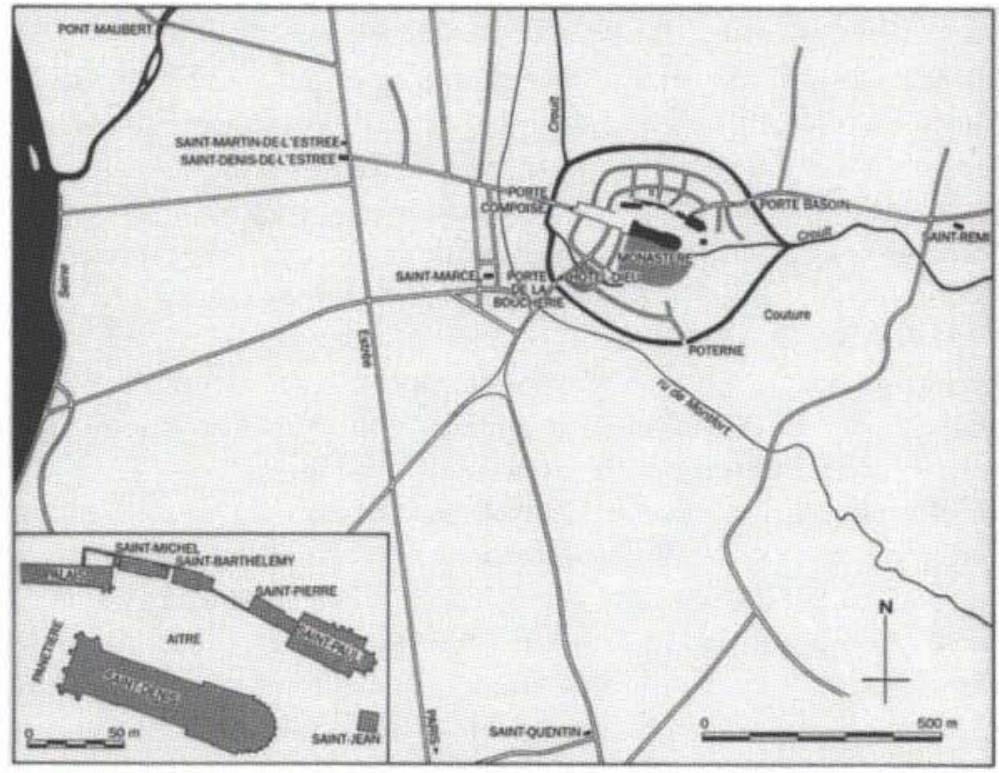

Fig. 1: Plan du bourg monastique et détail des abords du cimetière au XII siècle (dessin M. Wyss, UASD).

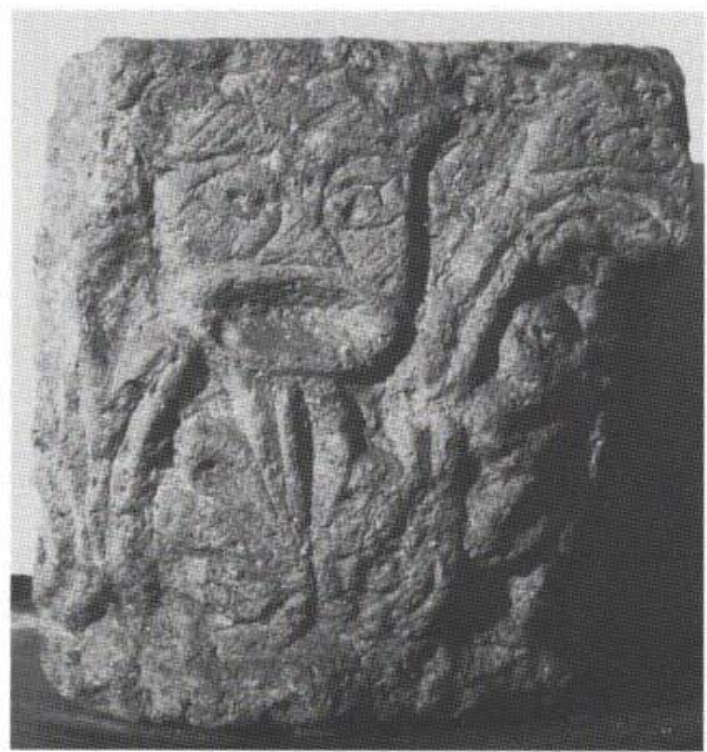

Fig. 2: Chapiteau à masque, deuxième moitié du $\mathrm{XI}^{\mathrm{e}}$ siècle (cliché J. Mangin, UASD). 


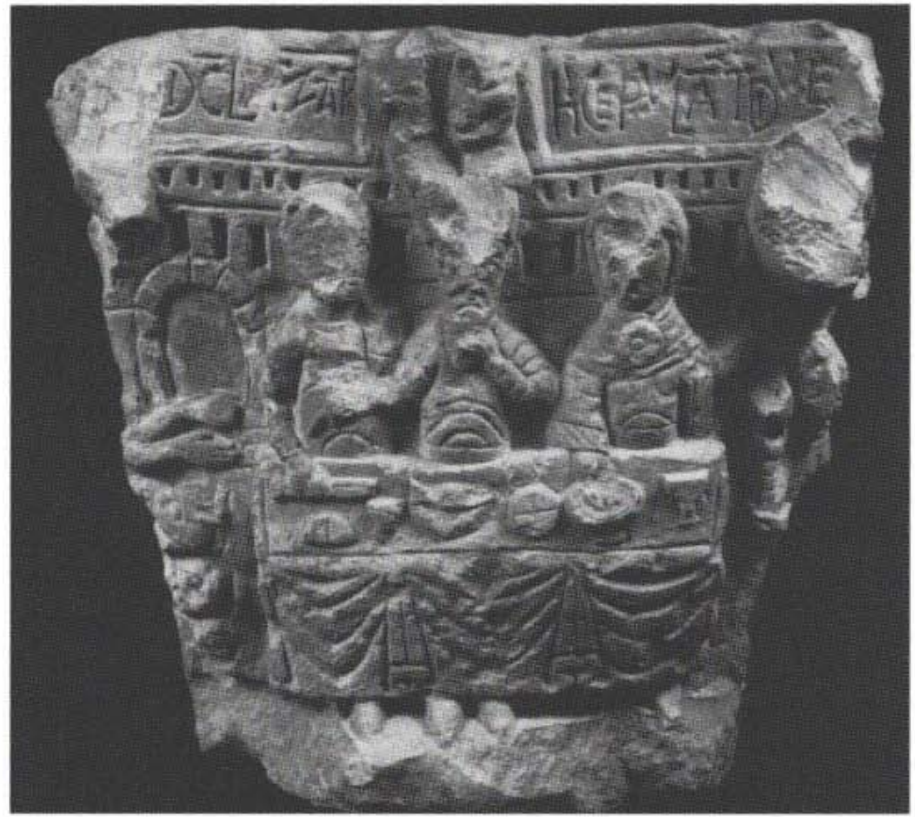

Fig. 3: Parabole du Mauvais Riche et de Lazare sur un chapiteau, vers 1120 (cliché E. Jacquot, UASD).

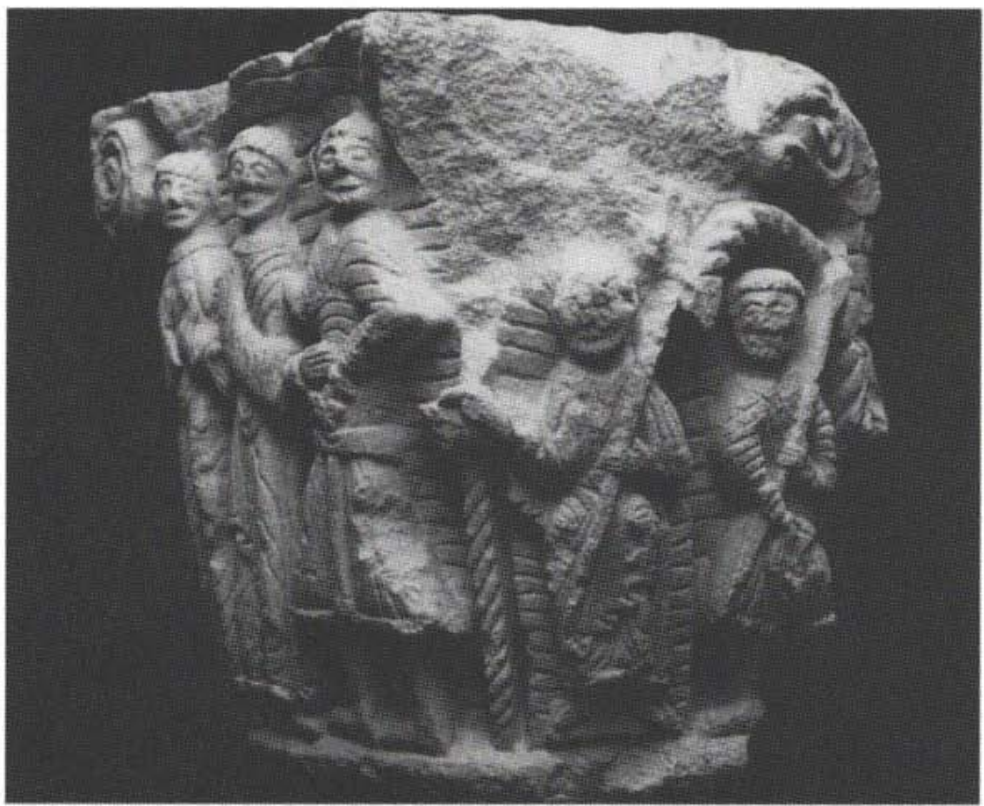

Fig. 4: Arrestation des trois saints Denis, Rustique et Éleuthère sur un chapiteau, vers 1120 (cliché E. Jacquot, UASD). 


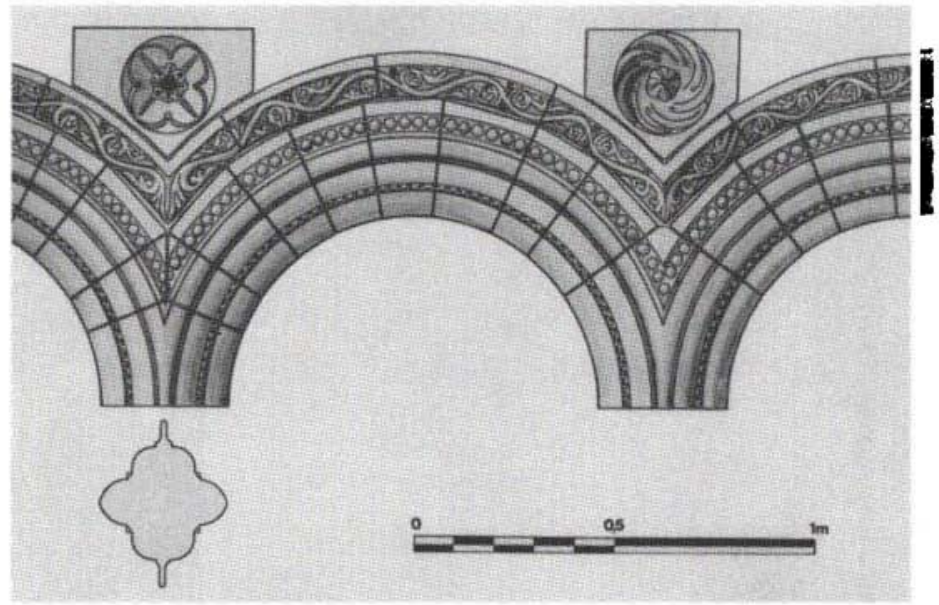

ilieu du

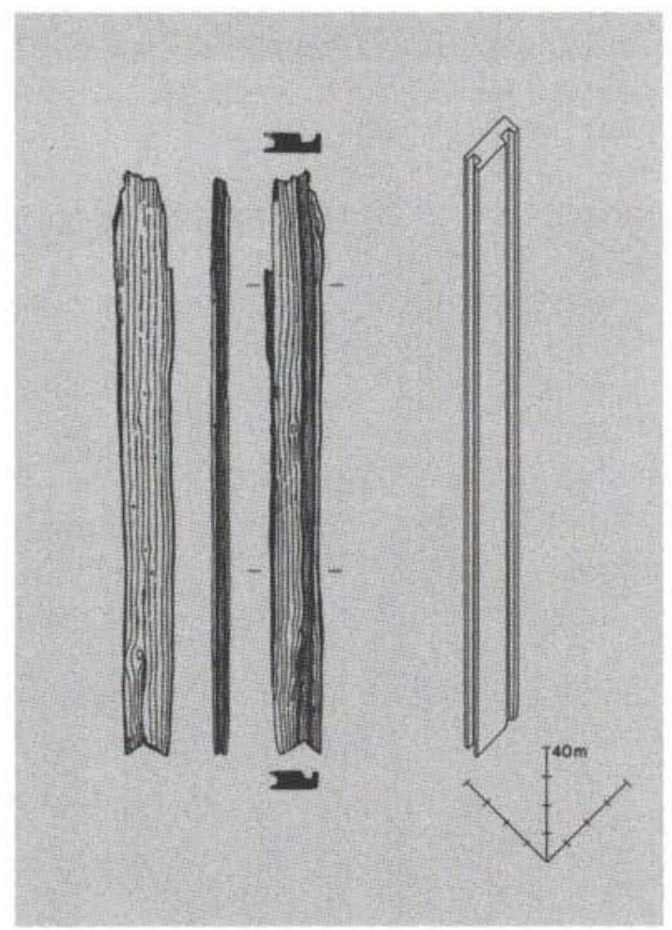

Fig. 6: Élément de Stabbau en chêne, XII ${ }^{e}$ siècle (dessin M. Wyss, UASD). 


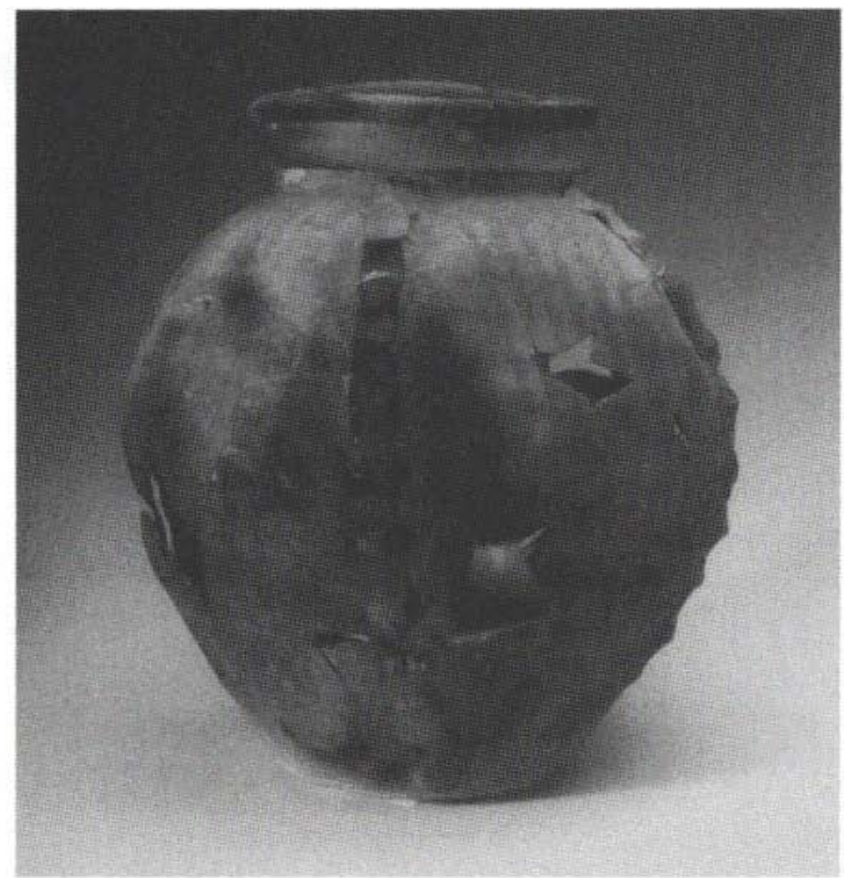

Fig. 7: Oule en céramique, $\mathrm{XII}^{e}$ siècle (cliché J. Mangin, UASD).

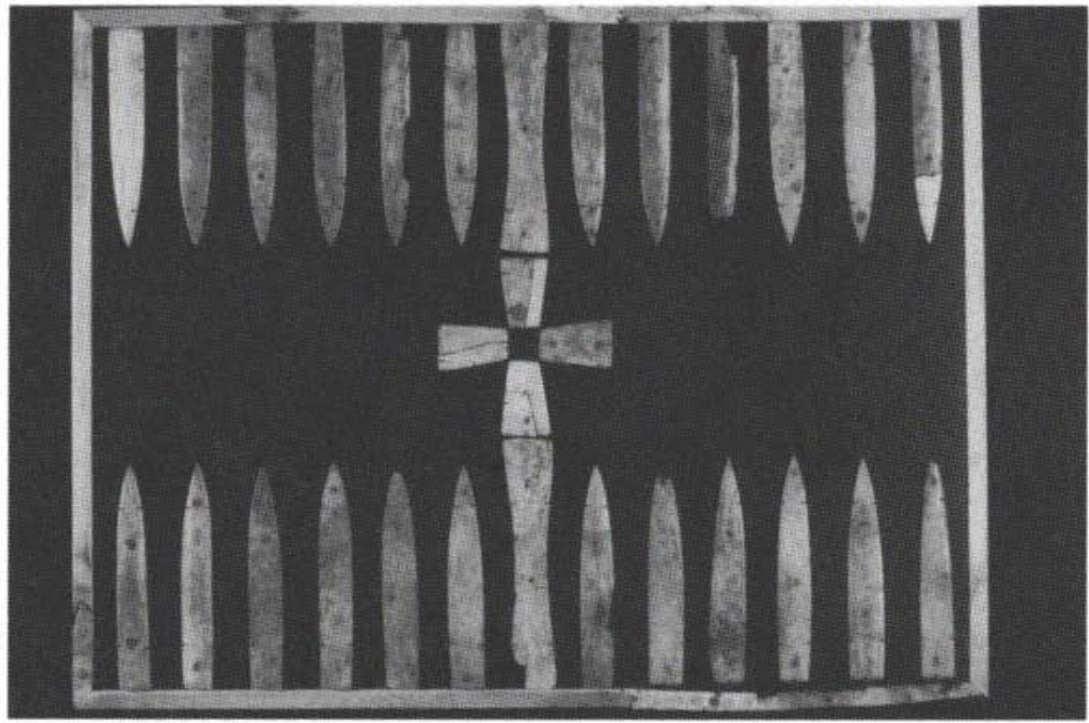

Fig. 8: Reconstitution d'un jeu de tables en os, XII' siècle (cliché E. Jacquot, UASD). 


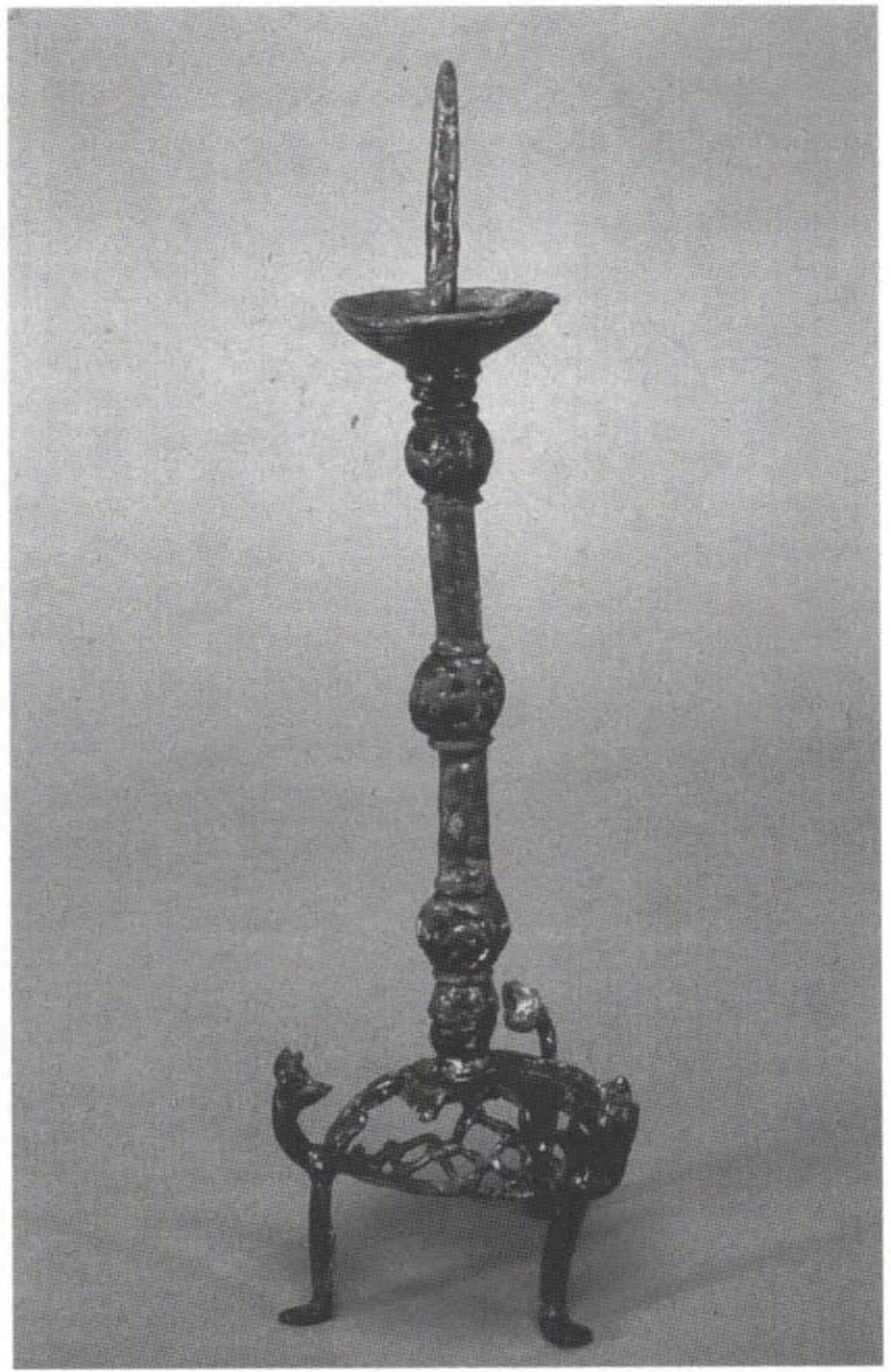

Fig. 9: Chandelier en bronze, $\mathrm{XI}^{e}$ siècle (cliché E. Jacquot, UASD). 